\title{
DETERMINANTES SOCIALES Y PERCEPCIÓN DE SALUD BUCAL DE LAS GESTANTES DEL HOSPITAL CIVIL DE TEPIC
}

\author{
Martínez-Mejía Vianey Jahayra1, Quiroga García Miguel Ángel ${ }^{2}$, Martínez González Gustavo Israel ${ }^{2}$, Aguiar \\ Fuentes Emma Genoveva ${ }^{3}$
}

1 Unidad Académica de Medicina - UAN 2 Universidad Autónoma de Nuevo León, Facultad de Odontología 3 Unidad Académica de Odontología - UAN

\section{RESUMEN}

Introducción: Los determinantes sociales según la OMS son las circunstancias en las que vivimos y desenvolvemos, repercuten directamente en la percepción de enfermedad y estado de salud poblacional. Identificarlos permite establecer estrategias para mejorar el estado de salud bucal y hábitos de control de placa, ya que es el pilar de prevención de principales enfermedades en boca a nivel mundial; enfermedad periodontal y caries, relevantes en gestación. Objetivo: Identificar determinantes sociales intermedios y estructurales de la población embarazada que acude a control, además de la relación existente con la percepción de la salud bucal. Métodos: Se realizó un estudio analítico, descriptivo transversal con la aplicación de una encuesta auto aplicada a 110 gestantes del hospital civil. El análisis se realizó en SPSS con la prueba estadística Xi2. Resultados: Existe relación estadística entre la percepción de salud bucal con 4 determinantes estructurales: etnia con una $p=.000$, la ocupación de la gestante $p=.035$, tipo de vivienda $p=0.029$ y con el servicio de drenaje $p=0.025$. Además de relacionarse estadísticamente con un DS intermedio: hábitos alimenticios (antojo de alimentos ácidos) con una $p=.004$. Conclusiones: La percepción de la salud bucal puede ser influenciada por determinantes sociales como los culturales, apego al tratamiento dental, algunos hábitos alimenticios, si pertenecen a alguna etnia, características de la vivienda y la ocupación de la gestante, sin embargo, aunque se identificaron creencias que pueden influir en la atención dental de la embarazada, pero sin significancia estadística. Se considera prudente realizar investigaciones cualitativas respecto a más factores intermedios.
\end{abstract}

Palabras Clave: determinantes sociales, percepción de salud oral, hábitos de higiene oral.

\section{ABSTRACT}

Introduction: The social determinants according to WHO are the circumstances in which we live and we directly affect the perception of diseases and health status in the population. Identifying allows you to set strategies to improve oral health status and habits in controlling plaque, as it's the pillar to prevent major diseases of the oral cavity worldwide; periodontal disease and caries, very important during pregnancy. Objective: To identify intermediary and structural social determinants of the pregnant population that comes to control, to the existing relationship with the perception of oral health. Methods: An analytical, descriptive cross-sectional study was carried out with the application of a self-administered survey to 110 pregnant women in the civil hospital. It was analyzed with statistical test Xi2 in SPSS. Results: there is a statistical relationship between the perception of oral health with four structural determinants: ethnicity with a $p=.000$, the pregnant mother's occupation $p=$ .035 , home $p=0.029$ and the drain service $p=0.025$. In addition to statistically related to an intermediary SD: eating habits (related to acid foods) with $p=.004$. Conclusions: The perception of oral health can be influenced by social determinants such as cultural, addiction to dental treatment, some eating habits, if they belong to some ethnic group, characteristics of the dwelling and occupation of the pregnant woman, however, although they were identified Beliefs that may influence the dental care of the pregnant woman, but without statistical significance. It is considered prudent to carry out qualitative research on more intermediate factors

Key words: social determinants, oral health perception, oral hygiene habits

Citation: Martínez-Mejía VJ, Quiroga-García MA, Martínez-González GI, Aguiar-Fuentes EG (2017) Determinantes sociales y percepción de salud bucal de las gestantes del Hospital Civil de Tepic. Revista de Salud Pública y Nutrición, 16(1), 16 - 22

Editor: Esteban G. Ramos Peña, Dr. CS., Universidad Autónoma de Nuevo león, Facultad de Salud Pública, Monterrey Nuevo León, México Copyright: ๑2017 Martínez-Mejía et al. This is an open-access article distributed under the terms of Creative Commons Attribution License [CC BY-ND 4.0], which permits unrestricted use, distribution, and reproduction in any medium, provided the original author and source are credited.

Competing interests: The authors have declared that no competing interests exist.

DOI: https://doi.org/10.29105/respyn16.1-3

Email: veyramia@hotmail.com 


\section{Introducción}

Los determinantes sociales de la salud (DSS) se describen por la Organización Mundial de la salud (OMS) como, "las circunstancias en las que las personas nacen, crecen, viven, trabajan y envejecen, incluido el sistema de salud". Descritas como el resultado de la distribución del dinero, el poder y los recursos a nivel mundial, nacional y local, que depende a su vez de las políticas adoptadas (Organización Mundial de la Salud, 2016). Se dividen en estructurales (factores estructurales; situación económica y política, entorno físico, normas y procesos de gobernanza) e intermedios (circunstancias de la vida diaria, exposición a factores que influyen en desarrollo de enfermedades) y repercuten directamente en el estado de salud (Organización Mundial de la Salud, 2008).

El establecimiento de un problema de salud es definido por la percepción del mismo y la utilización del sistema de salud (Sacchi, Hausberger, \& Pereyra, 2007). La percepción es definida como el conjunto de procesos cognitivos complejos que integran el estado interno y la relación con su entorno (Classe, 2011; Ramírez, y otros, 2015). Estudiar la percepción de la población, permite establecer y mejorar los programas de comunicación de riesgo sanitario (Stanojlovic, 2015), ya que, la cobertura y calidad de la atención médica están relacionados con el desarrollo del país, dónde la atención bucal adecuada es limitada, debido a sus altos costos (Padilla Loredo \& Cerón Argüelles, 2012). En México la caries y la enfermedad periodontal afectan a más del $50 \%$ de la población infantil y a más del $90 \%$ de la población adulta (Resultados del Sistema de Vigilancia Epidemiológica de Patologías Bucales (SIVEPAB) 2014, 2015).

La mayoría de los problemas que derivan en desigualdad o inequidad en salud están vinculados con los DSS (Palomino Moral, Grande Gascón, \& Linares, 2014) con diferentes efectos en cada grupo respecto las condiciones de vida cotidiana, a corto o largo plazo (The Interplay between Socioeconomic Inequalities and Clinical Oral Health, 2015), éstas disparidades son el resultado de la mala distribución de recursos y poder con gradientes sociales pronunciados, la alta carga de enfermedad necesita la implementación de políticas enérgicas a nivel macro con bases científicas dónde se comprometan tanto el gobierno como la población de todos los sectores (Lee \& Divaris, 2014; Sgan Cohen, y otros, 2013). Identificar los determinantes sociales en la población embarazada, permite informar, educar y motivar la atención adecuada en salud bucodental puesto que de ello depende que la gestante esté motivada a recibir el tratamiento, mejorar la salud bucal durante el embarazo o negarse a recibirlo por cuestiones culturales o creencias específicas como referir miedo a la atención dental o comprenderla como algo potencialmente dañino para el desarrollo fetal (Keirse \& Plutzer, 2010; Concha Sánchez, 2013). la OMS refiere que la salud bucodental no es solo la ausencia de dolor orofacial o patologías bucales, si no, a la limitación de actividades que repercuten en su bienestar psicosocial (Organización Mundial de la Salud, 2012).

La medida fundamental en odontología preventiva es el control de placa dentobacteriana en la higiene bucodental; precursora de la caries y la enfermedad periodontal, considerada como coadyuvante para la mejora de la salud bucal, mediante los métodos para controlar y eliminar la acumulación de placa como el uso de cepillo e hilo dental, cepillo interdental, pasta, clorhexidina y aceites esenciales (Higashida Hirose, 2009), estrategias que permitan la mejora de hábitos con el fin de un cambio en el comportamiento, estrategias educativas (Fernández Sedano, Unillos Landa, Zubieta, \& Rovira, 2004).

Durante el embarazo se experimentan cambios fisiológicos, hormonales y estructurales, clasificados como factores de riesgo biológicos, en dónde la salud bucal puede verse afectada por condiciones (factores ambientales) de la cavidad oral como el pH y la salivación (Martínez Pabón, Martínez, López Palacio, Patiño Gómez, \& Arango Pérez, 2014; Madianos, Bobetsis, \& Offenbacher, 2013), hábitos y costumbres adquiridos en éste periodo que representan factores de riesgo referentes a los estilos de vida y que pueden definir el curso de la enfermedad bucal (Murrieta Pruneda, López Rodríguez, Juárez López, Zurita Murillo, \& Linares Vieyra, 2013).

El estilo de vida tiene gran impacto en el cambio de actitudes, comportamientos, aspectos culturales y el acceso a la atención dental, pueden influir en la aceptación del tratamiento odontológico. La interacción de los DSS hacen vulnerable a la 
embarazada al desarrollo de focos de infección (Negroni, 1999; Corchuelo O, 2013), que de no ser detectados o eliminados oportunamente, pueden afectar su estado de salud general, así como el desarrollo y crecimiento fetal (de la Cruz Rodríguez \& de la Cruz Pérez, 2005; Herney \& Rengifo, 2009). Se han asociado al embarazo la enfermedad periodontal y el daño al esmalte relacionados con el vómito, inadecuada alimentación, higiene oral deficiente que aumenta la inflamación gingival y promueve el proceso carioso (Bauza, Martínez, Carmenate, Betancourt, \& García, 2016).

Existen barreras socioculturales que limitan la atención adecuada de la embarazada por miedo a la atención dental, baja percepción de riesgo y desinterés por su salud bucal (Fuentes, y otros, 2009), en una población embarazada estudiada en 2015, el principal motivo de consulta fue el dolor y el $66 \%$ no ha recibido atención dental (Krüger, y otros, 2015). Garibotti (2015), Cahuana, Palma, González y Palacios (2016) y cols coinciden en que hay una asociación entre los determinantes sociales presentes en las madres, como el nivel educativo y el nivel socioeconómico materno con resultados de salud desfavorables para ellas y sus fetos.

Los países de Latinoamérica y el Caribe comparten problemáticas de salud oral similares, sería prudente establecer estrategias de manera conjunta para determinar modelos curativos con menor inversión y mayor eficacia, dónde la atención al paciente sea holística, de manera que, se involucre el individuo, la sociedad, el personal de salud y la industria en pro del empoderamiento de la prevención y el cuidado de la salud bucal como una responsabilidad global (Contreras, 2016; Padilla Loredo \& Cerón Argüelles, 2012).

El objetivo de esta investigación fue identificar determinantes sociales en la población embarazada que acude a control, además de la relación existente con la percepción de la salud bucal.

\section{Material y Métodos}

Se realizó un estudio analítico, descriptivo transversal en el «Hospital Civil Dr. Antonio González Guevara». Se administró una encuesta auto aplicada a las embarazadas que aceptaron participar en el estudio, en caso de no hablar el idioma español se obtuvo ayuda de intérprete del mismo hospital o del acompañante. A cada participante se le informó de los objetivos de la investigación respaldado por el consentimiento informado.

Los aspectos éticos y legales fueron aprobados por el Comité de Bioética del mismo hospital.

La muestra fue calculada con un nivel de confianza de $95 \%$, ajustada a $15 \%$ de pérdidas, obteniendo el resultado de 110 participantes que fueron seleccionadas por un método no probabilístico de tipo casual. Se incluyeron las embarazadas que acudieron a control prenatal o al módulo mater en el "Hospital Civil Dr. Antonio González Guevara" y desearon participar; se eliminaron encuestas incompletas.

Para el análisis estadístico se utilizó el programa SPSS con la prueba de $\mathrm{Xi}^{2}$.

Resultados

La población estudiada con un total de 110 participantes, tuvo una distribución entre los 14 y 42 años de edad con una media de $2416 \pm 6.975$ años; de las cuales, $28.2 \%$ son adolescentes y $9.9 \%$ son pacientes añosas.

Las variables analizadas corresponden a los DSS estructurales e intermedios y su relación con la percepción de salud bucal.

Los DSS estructurales estudiados fueron: zona de procedencia, etnia, escolaridad de la gestante y de su pareja, la ocupación de la gestante y de la pareja y quién funge como jefe de familia, tipo y servicios de la vivienda. Cuatro de los determinantes sociales estructurales estuvieron asociados a la percepción de salud bucal (Tabla 1).

De los DS intermedios: tipo de embarazo, número de embarazos, edad gestacional, creencias, si recibió información y atención dental prenatal, hábitos de higiene bucal, afecciones dentales y hábitos alimenticios, solo en el de consumo de alimentos ácidos se encontró asociación con la percepción de salud bucal (Tabla 2). 
Tabla 2. Población gestante del Hospital Civil «Dr.

González Guevara»según Determinantes

Sociales Intermedios identificados

Tabla 1. Población gestante del Hospital Civil «Dr. González Guevara»según Determinantes Sociales Estructurales identificados

\begin{tabular}{|c|c|}
\hline Determinante Social & $\%$ \\
\hline \multicolumn{2}{|l|}{ Zona de procedencia } \\
\hline Urbana & 63.6 \\
\hline Rural & 36.4 \\
\hline \multicolumn{2}{|l|}{ Pertenencia a etnia } \\
\hline si & 18.1 \\
\hline no & 81.9 \\
\hline \multicolumn{2}{|l|}{ Ocupación (gestante) } \\
\hline Lona rura (ama ae casa) & $0<.0$ \\
\hline Zona Urbana (ama de casa) & 35.7 \\
\hline \multicolumn{2}{|l|}{ Ocupación (pareja) } \\
\hline \multicolumn{2}{|l|}{ Zona rural } \\
\hline (agricultura/ganadería) & 45.0 \\
\hline Zona Urbana (estudiante / otra) & 41.4 \\
\hline \multicolumn{2}{|l|}{ Escolaridad (gestante) } \\
\hline Zona rural (primaria) & 30.0 \\
\hline Zona Urbana (secundaria) & 41.4 \\
\hline \multicolumn{2}{|l|}{ Escolaridad (pareja) } \\
\hline Zona rural (primaria) & 37.5 \\
\hline Zona Urbana (secundaria) & 38.6 \\
\hline \multicolumn{2}{|l|}{ Jefe de familia } \\
\hline Pareja & 52.2 \\
\hline Entre lo dos & 34.5 \\
\hline \multicolumn{2}{|l|}{ Tipo de vivienda } \\
\hline rropıa & 41.3 \\
\hline Rentada & 23.6 \\
\hline Prestada & 24.5 \\
\hline \multicolumn{2}{|l|}{ Servicios de la vivienda } \\
\hline Agua potable & 97.3 \\
\hline Luz & 97.3 \\
\hline Drenaje & 84.5 \\
\hline
\end{tabular}

Fuente: Elaboración propia; resultados de la encuesta a gestantes del Hospital Civil «Dr. González Guevara»

Determinante Social $\quad \%$

Tipo de embarazo

Adolescentes 11.8

$\begin{array}{cc}\text { Alto riesgo } & 32.7 \\ & 55.5\end{array}$

Normal $\quad 55.5$

Edad gestacional (trimestre)

Primer 27.3

Segundo $\quad 30.9$

Tercer $\quad 41.8$

Número de embarazos

Primigestas $\quad 57.3$

Multigestas $\quad 42.7$

Información de salud bucal

No recipıo su.y

SI recipio $\quad 49.4$

Atención bucal

No recibió $\quad 60.9$

Si recibió $\quad 39.1$

Hábitos de higiene bucal

Utilizan pasta dental $\quad 93.6$

$\begin{array}{ll}\text { Utilizan pasta dental } & 93.6 \\ \text { UtIIIzan enjuage pucal } & 34.5\end{array}$

Utilizan hilo dental $\quad 21.8$

Lavan tres veces al día $\quad 44.5$

Afecciones dentales

Inflamación gingival $\quad 35.5$

Sangrado gingival $\quad 30.9$

Sensibilidad dental $\quad 23.6$

Dolo dental $\quad 20.9$

Hábitos alimenticios (antojos)

Hielo $\quad 35.5$

Chile y limón $\quad 34.5$

Alımentos acıaos" 33.0

Uuices y chocolates 25.5

Pastel 24.5

comer tierra $\quad 8.2$

Creencias (durante el embarazo)

No debe ir al dentista 20

Se produce caries $\quad 49$

Los dientes se caen $\quad 39$

Es normal se caiqan los dientes $\quad 34.5$

Se produce sengrado de encias $\quad 29$

Es normal que sangren las encias 26

Lavarse los dientes afecta al bebe $\quad 9$

Tener miedo ir al dentista $\quad 39$

Fuente: Elaboración propia; resultados de la encuesta a gestantes del Hospital Civil «Dr. González Guevara» $p<0.01$ en relación con percepción de salud bucal 


\section{Discusión}

Se han realizado estudios en los que se afirma una asociación con el desapego a los tratamientos dentales durante el embarazo por creencias y costumbres de la gestantes (Concha Sánchez, 2013).

Los determinantes relacionados con las creencias pueden influenciar la decisión de las embarazadas a recibir o negar la atención odontológica durante el embarazo, al identificarse respuestas como "temor al dentista", "es normal perder diente durante el embarazo", y "el embarazo produce caries".

Fuentes y cols. (2009) Identificaron que el 22\% de una población de 65 gestantes, refiere no haber buscado tratamiento dental durante el embarazo por temor.

Otros determinantes estructurales como la escolaridad, referida por Fernández, y cols. (2004), especialmente en zonas rurales dónde el nivel de estudios tiende a ser menor a la preparatoria y pueden asociarse con las creencias negativas respecto a los tratamientos dentales y sus efectos en el desarrollo fetal por la falta de atención odontológica, influenciando la percepción bucodental.

Con fines de mejoras en el tema, es importante promover una higiene bucal eficiente (Bouza) y al mismo tiempo definir (priorizar) el motivo de consulta de la gestante (Garibotti), ya que durante el embarazo el principal motivo de consulta es por dolor y no por prevención.

Como lo menciona Garibotti y colaboradores (2015), es necesario definir el motivo de consulta de las gestantes y promover las higiene bucal eficiente según Bouza Vera y cols (2016), ya que durante el embarazo la cavidad bucal de la gestante tiene modificaciones que la hacen susceptible a patologías bucales y el mejor coadyuvante es la mejora en la higiene bucal (Higashida Hirose, 2009).

\section{Conclusiones}

La percepción de la salud bucal de las gestantes es influenciada por varios determinantes, especialmente culturales, como creencias, mitos, costumbres y miedos respecto al tratamiento buco-dental, debido a una carencia o mala información.
Los resultados obtenidos coinciden con lo publicado por Concha Sánchez (2013); se identificaron creencias que pueden influir en la decisión de la gestante respecto a la atención durante el embarazo; tales como, referir temor a la atención dental; de la población estudiada (110 gestantes) 39\%, menos de la mitad de la población encuestada, lavan sus dientes por lo menos 3 veces al día y $35.5 \%$ refirieron sangrado gingival, sin embargo, sólo $39.1 \%$ recibió atención dental.

Aunque, el nivel de percepción fue identificado, en su mayoría como regular (46.4\%), el tratamiento bucodental es prioritario en embarazos de alto riesgo, adolescentes y en pacientes añosas (mayores a 35 años); de las mujeres encuestadas, el $28.2 \%$ son adolescentes y $9.9 \%$ son pacientes mayores a 35 años, se considera prudente establecer estrategias dirigidas a dichos grupos oportunamente.

El nivel de estudios está relacionado con la asistencia y el apego al tratamiento, a su vez se relacionan con la ocupación de la gestante, la zona de procedencia y el rol del jefe de familia; más acentuado en la zona rural. Se determinó relación estadística significativa $\left(\mathrm{Xi}^{2}\right)$ entre la percepción de salud bucal y la etnia con una $\mathrm{p}$ igual a .000 , la ocupación de la gestante con una $p$ de 0.035. por ello, se puede inferir la necesidad de instrucción y educación respecto al tema de salud bucal en el embarazo, especialmente en zonas rurales y en gestantes pertenecientes a una etnia.

Por último, se cree prudente analizar el estado socioeconómico de las gestantes ya que se obtuvo relación estadística $(\mathrm{p}=.029)$ con el tipo de vivienda y servicios como drenaje con la percepción de salud oral $(\mathrm{p}=0.025)$. Ya qué, las familias que no cuentan con los servicios básicos y una vivienda propia, pudieran tener condiciones más desfavorables para tener una salud bucal favorable durante el embarazo.

\footnotetext{
Agradecimientos:

Al personal del Hospital Civil "Dr. González Guevara" por permitirme realizar el estudio en sus instalaciones, a las pacientes que me brindaron su ayuda, a los doctores y maestros de la Universidad Autónoma de Nayarit y de la Universidad Autónoma de Nuevo León por su invaluable colaboración en la presente investigación.
} 


\section{Bibliografía}

Bauza, M., Martínez, J., Carmenate, Y., Betancourt, M., \& García, M. (2016). Pregnancy and oral health. Rev Med Electrón, 38(4), 628-34. Recuperado el 22 de septiembre de 2016, de http://scielo.sld.cu/scielo.php?script=sci_arttext\&pid=S16 84-18242016000400013\&lng=es

Cahuana, A., Palma, C., González, Y., \& Palacios, E. (2016). Maternal and infant oral health care. How do we improve it? Matronas Prof, 17(1), 12-9. Recuperado el 22 de septiembre de 2016, de http://www.federacionmatronas.org/rs/1405/d112d6ad-54ec-438b-93584483f9e98868/e83/fd/1/filename/articulo-especialsalud-bucodental.pdf.

Classe, Q. S. (2011). Enciclopediasalud.com. Recuperado el 19 de septiembre de 2016, de http://www.enciclopediasalud.com/definiciones/percepció n

Concha Sánchez, S. C. (2013). El proceso salud-enfermedadatención bucal de la gestante: una visión de las mujeres con base en la determinación social de la salud. Rev Fac Med Univ Nac Colomb, 61(3), 275-91. Recuperado el 15 de octubre de 2016, de http://www.scielo.org.co/pdf/rfmun/v61n3/v61n3a08

Contreras, A. (2016). La promoción de la salud general y la salud oral: una estrategia conjunta. Rev Clínica Periodoncia, Implantol y Rehabil Oral, 9(2), 193-202. Recuperado el 20 de septiembre de 2016, de http://dx.doi.org/10.1016/j.piro.2016.07.003

Corchuelo O, J. (2013). Determinantes sociales y del estilo de vida en salud oral en el acceso a odontología de gestantes caleñas en el 2012. Rev Fac Nac Salud Pública, 31(1), 170180. Recuperado el 13 de febrero de 2017, de http://www.scielo.org.co/scielo.php?script=sci arttext\&pi $\mathrm{d}=\mathrm{S} 0120-386 \mathrm{X} 2013000400019$

de la Cruz Rodríguez, A., \& de la Cruz Pérez, M. (2005). Ciencias de la salud: una aproximación vivencial. México, D. F.: McGraw-Hill. Recuperado el 20 de octubre de 2016

Fernández Sedano, I., Unillos Landa, S., Zubieta, E., \& Rovira. (2004). Psicología social, cultura y educación. España: Pearson Educación. Recuperado el 13 de septiembre de 2016

Fuentes, R., Oporto, G., Alarcón, A., Bustos, L., Prieto, R., \& Rico, H. (2009). Opiniones y creencias de las embarazadas en control prenatal relacionadas con la salud oral y embarazo. Av Odontoestomatol, 25(3), 147-54. Recuperado el 22 de septiembre de 2016, de http://scielo.isciii.es/pdf/odonto/v25n3/original3.pdf
Garibotti, G., Vasconi, C., Ferrari, A., Giannini, G., Colmar, H., \& Schnaiderman, D. (2015). Percepción parental de la salud psicofísica, estado nutricional y salud bucal, en relación con las características sociodemográficas en niños de Bariloche, Argentina: estudio epidemiológico. Arch Argent Pediatr, 113(5), 411-8. Recuperado el 21 de septiembre de 2016, de http://www.sap.org.ar/docs/publicaciones/archivosarg/201 5/v113n5a06.pdf

Herney, A., \& Rengifo, R. (2009). Beliefs about the oral health in pregnant women in three colombian cities. Rev Fac Odontol Univ Antioq, 20(2), 171-8. Recuperado el 25 de septiembre de 2016, de http://www.scielo.org.co/pdf/rfoua/v20n2/v20n2a08.pdf

Higashida Hirose, B. Y. (2009). Odontología preventiva (segunda edición ed.). México: McGraw-Hill. Recuperado el 15 de octubre de 2016

Keirse, M., \& Plutzer, K. (2010). Women's attitudes to and perceptions of oral health and dental care during pregnancy. J Perinat Med, 38(1), 3-8. Recuperado el 8 de octubre de 2016, de http://edoc.huberlin.de/oa/degruyter/jpm.2010.007.pdf

Krüger, S. M., Lang, C. A., Almeida, H. S., Bello, F. O., Romano, A. R., \& Pappen, F. G. (2015). Dental pain and associated factors among pregnant women: An observational study. Matern Child Health J, 19, 504-10. Recuperado el 22 de septiembre de 2016, de http://aleph.uan.edu.mx:2093/static/pdf/239/art:10.1007/s 10995-014-1531y.pdf?originUrl=http://link.springer.com/article/10.1007/s 10995-014-1531$\mathrm{y} \&$ token $2=\exp =1458023310 \sim \mathrm{acl}=/ \mathrm{static} / \mathrm{pdf} / 239 / \mathrm{art} \% 3 \mathrm{~A}$ $10.1007 \% 2 F s 10995-014-153$

Lee, J. Y., \& Divaris, K. (2014). The Ethical Imperative of Addressing Oral Health Disparities: A Unifyng Framework. J Dent Res, 93(3), 224-230. doi:10.1177/0022034513511821

Madianos, P. N., Bobetsis, Y. A., \& Offenbacher, S. (2013). Adverse pregnancy outcomes (APOs) and periodontal disease: Pathogenic mechanisms. J Clin Periodontol, 40(14), 170-80. Recuperado el 20 de octubre de 2016, de http://www.joponline.org/doi/pdf/10.1902/jop.2013.1340 015

Martínez Pabón, M., Martínez, C., López Palacio, A., Patiño Gómez, L., \& Arango Pérez, E. (2014). Caracterìsticas fisicoquìmicas y microbiològicas de la saliva durante $\mathrm{y}$ despuès del embarazo. Rev Salud Pùblica, 16(1), 128-38. Obtenido de http://www.redalyc.org/articulo.oa?id=42232032011 
Murrieta Pruneda, J. F., López Rodríguez, Y., Juárez López, L. A., Zurita Murillo, V., \& Linares Vieyra, C. (2013). Indices epidemiológicos de morbilidad bucal. Universidad Nacional Autónoma de México: Facultad de Estudios Superiores "Zaragoza", 192. Recuperado el 20 de septiembre de 2016, de flap.com/oqRFCm7iGGgrKoplky9sbq\#.V7PrrPnhDIU=\& $\mathrm{p}=0$

Negroni, M. (1999). Microbiología estomatológica. Argentina: Editorial Médica Panamericana. Recuperado el 20 de octubre de 2016

Organización Mundial de la Salud. (2008). Informe final de la Comisión sobre Determinantes Sociales de la Salud OMS. Ginebra, Suiza: Biblioteca de la OMS. Recuperado el 19 de septiembre de 2016, de http://www.who.int/social_determinants/final_report/med ia/csdh_report_wrs_es.pdf

Organización Mundial de la Salud. (2012). Nota informativa $N^{\circ} 318$; salud bucodental. Recuperado el 23 de septiembre de 2016, de http://www.who.int/mediacentre/factsheets/fs318/es/

Organización Mundial de la Salud. (2016). Organización Mundial de la Salud. Obtenido de http://www.who.int/social determinants/es/

Padilla Loredo, S., \& Cerón Argüelles, J. (agosto de 2012). Determinantes culturales, económicos, históricos, políticos y sociales de la salud bucal. Medware, 12(7). doi:10.5867/medwave.2012.07.5452

Palomino Moral, P. A., Grande Gascón, M. L., \& Linares, A. M. (2014). La salud y sus determinantes sociales; Desigualdades y exclusión en la sociedad del siglo XXI. Rev Int Sociol, 72(1), 45-70. Recuperado el 15 de Agosto de 2016, de http://dialnet.unirioja.es/servlet/articulo?codigo $=4894610$ \&info=resumen\&idioma $=$ SPA

Ramírez, M., Suárez Paniagua, S., de la Fuente Hernández, J., Shimada Beltrán, H., Reyes Durán, J. F., \& Acosta Torres, L. S. (2015). Componentes culturales que influyen en la salud bucal. Salud(i)ciencia, 21(3), 294-300. Recuperado el 19 de septiembre de 2016, de https://www.siicsalud.com/dato/sic/213/139635.pdf

Secretaría de Salud. (2015). Resultados del Sistema de Vigilancia Epidemiológica de Patologías Bucales (SIVEPAB) 2014. Ciudad de México: Secretaría de Salud. Recuperado el 13 de febrero de 2017, de http://www.epidemiologia.salud.gob.mx/doctos/infoepid/ bol_sivepab/SIVEPAB-2014.pdf
Sacchi, M., Hausberger, M., \& Pereyra, A. (2007). Percepción del proceso salud-enfermedad-atención y aspectos que influyen en la baja utilización del Sistema de Salud, en familias pobres de la ciudad de Salta. Salud Colect, 3(3), 27183. Recuperado el 19 de septiembre de 2016, de http://www.scielo.org.ar/scielo.php?script=sci_arttext\&pi $\mathrm{d}=\mathrm{S} 1851-82652007000300005$

Sgan Cohen, H. D., Evans, R. W., Whelton, H., Villena, R. S., MacDougall, M., Williams, D. M., \& IADR-Gohira Steering and Task Groups. (2013). IADR Global Oral health Inequialities Research Agenda (IADR-GOHIRA): A Call to Action. J Dent Res, 92(3), 209-2011. doi: $10.1177 / 0022034512475214$

Stanojlovic, M. (2015). Percepción social de riesgo: una mirada general y aplicación a la comunicación de salud. Rev Comun y Salud, 5, 96-107. Recuperado el 15 de septiembre de 2016, de http://www.revistadecomunicacionysalud.org/index.php/r cys/article/view/83

The Interplay between Socioeconomic Inequalities and Clinical Oral Health. (2015). Journal of Dental Research, 94(1), 19-26. doi:10.1177/0022034514553978 